Original Paper http://ajol.info/index.php/ijbcs http://indexmedicus.afro.who.int

\title{
Phytochimie, activité antioxydante et toxicité aiguë de plantes médicinales utilisées contre les séquelles de l'accident vasculaire cérébral en Côte d'Ivoire
}

\author{
Serge Cherry PIBA ${ }^{1 *}$, Pierre Alain Kouassi KONAN ${ }^{2}$, Losséni N'gana KONE ${ }^{3}$, Amoin \\ Gervaise KOUAME ${ }^{4}$, Richard Kouakoubah Donthy KOUAKOU ${ }^{5}$ et \\ Honora Fezan Bi TRA ${ }^{6}$
}

1 Université de Man UFR Ingénierie Agronomique Forestière et Environnementale. Université Nangui Abrogoua, Laboratoire de Chimie Bioorganique des Substances Naturelles. Université Nangui Abrogoua, UFR Sciences de la Nature.

\footnotetext{
2 Université Nangui Abrogoua, UFR Sciences de la Nature.

3 Université Nangui Abrogoua, UFR Sciences de la Nature.

${ }^{4}$ Université Nangui Abrogoua, UFR Sciences de la Nature.

*Auteur correspondant, E-mail: serge.piba@univ-man.edu.ci
}

\section{REMERCIEMENTS}

Les auteurs de ce manuscrit remercient l'ASCAD (Académie des Sciences, des Arts, des Cultures d'Afrique et des Diasporas Africaines) pour sa contribution financière à cette étude.

\begin{tabular}{ccc}
\hline Received: 08-10-2020 & Accepted: 29-01-2021 & Published: 30-04-2021 \\
\hline
\end{tabular}

\section{RESUME}

Les séquelles, après un accident vasculaire cérébral, sont importantes et les patients handicapés moteurs très nombreux. La présente étude a pour objectif de définir le profil phytochimique des cinq plantes les plus utilisées pour traiter les séquelles des AVC en Côte d'Ivoire, d'évaluer leur capacité antioxydante et leur toxicité. Après le screening phytochimique, la teneur en phénols et flavonoïdes ont été quantifiés par la méthode colorimétrique de Folin-Ciocalteu. L'évaluation quantitative du potentiel antioxydant des extraits a été réalisée in vitro par le test au DPPH (1,1-diphényl-2-picryl-hydrazyle). Les tests ont mis en évidence la présence de polyphénols, de tanins et de flavonoïdes dans tous les extraits aqueux. Ces métabolites sont importants dans la prévention et le traitement des maladies cardio-vasculaires. Les taux les plus élevés de polyphénols sont présents dans les extraits aqueux d'écorce de tige de Ficus mucuso (83425,29 $\mu \mathrm{g}$ EAG/g MS) et de rameaux feuillés de Zizyphus mauritiana (74517,24 $\mu \mathrm{g}$ EAG/g MS). La plus forte teneur en flavonoïdes a été observée dans l'extrait aqueux de rameaux feuillés de Ziziphus mauritiana (20,28\%). Des capacités antioxydantes importantes ont été observées dans les extraits aqueux du mélange écorces de tige/feuilles de Tamarindus indica $\left(\mathrm{Cl}_{50}=0,1269\right.$ $\mathrm{mg} / \mathrm{ml}$ ) et dans l'extrait d'écorce de tige de Ficus mucuso $(0,1335 \mathrm{mg} / \mathrm{ml})$. Les tests de toxicité aigüe montrent que l'extrait aqueux de l'écorce de tige de Ficus mucuso, par voie orale, à $5000 \mathrm{mg} / \mathrm{kg}$ de pc, est légèrement toxique chez les souris.

() 2021 International Formulae Group. All rights reserved.

Mots clés : AVC, séquelles, plantes médicinales, phytochimie, toxicité. 


\title{
Phytochemistry, antioxidant activity and acute toxicity of medicinal plants used against the sequelae of stroke in Côte d'Ivoire
}

\begin{abstract}
The sequelae after a stroke are significant and there are many patients with motor disabilities. The purpose of this study is to define the phytochemical profile of the five plants most used to treat the sequelae of stroke in Côte d'Ivoire, to assess their antioxidant capacity and their toxicity. After the phytochemical screening, the content of phenols and flavonoids were quantified by the colorimetric method of Folin-Ciocalteu. The quantitative evaluation of the antioxidant potential of the extracts was carried out in vitro by the DPPH $(1,1-$ diphenyl-2-picryl-hydrazyl) test. The tests revealed the presence of polyphenols, tannins and flavonoids in all the aqueous extracts. These metabolites are important in the prevention and treatment of cardiovascular disease. The highest levels of polyphenols are found in aqueous extracts of stem bark of Ficus mucuso (83425.29 $\mu \mathrm{g}$ EAG/ g DM) and leafy twigs of Zizyphus mauritiana (74,517.24 $\mu \mathrm{g}$ EAG / g DM). The highest content of flavonoids was observed in the aqueous extract of leafy twigs of Ziziphus mauritiana (20.28\%). Significant antioxidant capacities were observed in the aqueous extracts of the stem bark / leaf mixture of Tamarindus indica (IC50 $=0.1269 \mathrm{mg} / \mathrm{ml}$ ) and in the extract of the stem bark of Ficus mucuso $(0.1335 \mathrm{mg} / \mathrm{ml})$. Acute toxicity tests show that the aqueous extract of the stem bark of Ficus mucuso, taken orally, at $5000 \mathrm{mg} / \mathrm{kg}$ bw, is slightly toxic in mice.
\end{abstract}

(C) 2021 International Formulae Group. All rights reserved.

Keywords: Stroke, sequelae, medicinal plants, phytochemistry, toxicity.

\section{INTRODUCTION}

Les Accidents Vasculaires cérébraux (AVC) sont la cause de nombreux décès dans le monde et la première cause de handicap moteur chez l'adulte (Feigin et al., 2014 ; Go et al., 2014). La thrombolyse est, aujourd'hui, le traitement de référence en cas de survenu d'une ischémie cérébrale (Bordet et al., 2009 ; Leys et al., 2009 ; Lamblin et al., 2018) responsable de $85 \%$ des AVC. Cependant, pour diverses raisons, elle ne peut être réalisée que sur quelques patients (Leys et al., 2009 ; Masingue et Alamowitch, 2014 ; Quintard, 2014). Une des limites réside dans cette fenêtre thérapeutique étroite car, au-delà du délai recommandé (4h30 $\mathrm{min})$, le risque hémorragique est élevé (Abboud et Amarenco, 2004 ; Hacke et al., 2008). Les séquelles après la survenue des AVC sont donc importantes et les patients handicapés moteurs très nombreux (HAS, 2015). L'atteinte neurologique est souvent très grande et le traitement des séquelles invalidantes reste mal maitrisé (HAS, 2015). Dans la prise en charge post AVC, les résultats des essais thérapeutiques par la neuroprotection, la neurogenèse puis l'utilisation de cellule souche, restent mitigés (Sutherland et al., 2012). Pour aider à la réadaptation de la fonction motrice, la mise en œuvre d'une rééducation adaptée nécessite une évaluation rapide par des professionnels de la rééducation tels que le masseur kinésithérapeute, l'orthophoniste, l'ergothérapeute, etc. (HAS, 2012). Dans les pays tropicaux, où le déficit en personnel de santé qualifié et en infrastructures cliniques adéquates est important et limite la prise en charge des malades, l'utilisation des plantes dans le traitement des séquelles des AVC reste l'une des alternatives pour les populations pauvres et démunies. La phytothérapie dans le traitement et la prévention des maladies cardiovasculaires est reconnue (Kee et al., 2008 ; Luna-Vázquez et al., 2013). De récentes 
études ont montré que les tanins présentent des propriétés anticoagulantes (Kee et al., 2008), certaines polyphénols présentent des propriétés vasodilatatrices et antihypertensives (Proteggente et al., 2011). Les antioxydants, très recherchés pour leur valorisation dans de nombreux pays (Agbodan et al., 2014 ; Evenamede et al., 2017), contribuent à la réduction du stress oxydatif lié à la survenue de la lésion cérébrale et favorisent la régénération cellulaire (Quintard, 2014). En Côte d'Ivoire, de nombreuses drogues végétales sont vendues sur les marchés et utilisées dans le traitement des facteurs des risques (hypertension, diabète) et des séquelles des AVC telles que l'hémiplégie et l'aphasie (Piba et al., 2018). Parmi ces drogues, les écorces de tige de Ficus mисиso, les rameaux feuillés de Ziziphus mauritiana, la plante entière de Catharantus roseus, les feuilles de Ocimum graticimum, le mélange écorces de tige/feuilles de Tamarindus indica sont les plus utilisées (Piba et al., 2018). L'objectif de cette étude est de définir le profil phytochimique de ces drogues végétales fréquemment utilisées, leur capacité antioxydante et d'évaluer leur toxicité, conformément aux thérapies définies par les herboristes pour une meilleure prise en charge des malades victimes des AVC.

\section{MATERIEL ET METHODES}

\section{Préparations des extraits bruts}

Le protocole d'extraction était le plus proche possible des indications des herboristes pour être conforme à leur mode de préparations. La première étape de ce processus était la préparation par décoction des extraits aqueux. Les différents organes de plantes sélectionnées (Tableau 1) ont été collectés auprès des herboristes, dans les marchés. Ils ont été nettoyés, découpés en petits morceaux, séchés dans une salle sous climatisation à $18 \pm 2{ }^{\circ} \mathrm{C}$, puis pulvérisés à l'aide d'un broyeur électrique. Pour chaque organe, $100 \mathrm{~g}$ de poudre végétale fine ont été dissouts dans 2 litres d'eau distillée et l'ensemble porté à ébullition pendant $45 \mathrm{~min}$. Le décocté a été filtré sous vide à l'aide d'un entonnoir contenant du coton et du papier filtre pour obtenir un (1) litre de filtrat. Les solutions obtenues ont été directement lyophilisées par un lyophilisateur CHRIST, ALPHA 2-4 LO. Les différents extraits bruts (lyophilisats) ont, ensuite, été pesés et stockés dans un récipient étanche et conservés à température constante (4 $\left.{ }^{\circ} \mathrm{C}\right)$.

\section{Criblage phytochimique}

Le criblage phytochimique a été réalisé suivant les procédures analytiques décrites dans les travaux de (Harbome et al., 1998). Les polyphénols et les tanins sont mis en évidence par la réaction au chlorure ferrique $\left(\mathrm{FeCl}_{3}\right)$. Les flavonoïdes sont détectés au moyen de la réaction au trichlorure d'aluminium et les stérols à l'aide de l'acide sulfurique concentré $\left(\mathrm{H}_{2} \mathrm{SO}_{4}\right)$, puis les alcaloïdes à partir du réactif de Dragendor'ff et les saponosides par le test de la mousse. Ces différents groupes de composés chimiques ont été retenus pour leur importance dans le traitement et la prévention des AVC (Ramya et al., 2007 ; Kee et al., 2008).

\section{Dosage des composés phénoliques totaux et des flavonoïdes}

La quantité de phénols totaux des extraits de végétaux bruts a été déterminée par la méthode colorimétrique de Folin-Ciocalteu selon Singleton et al. (1999) et Heilerová et al. (2003). Le mélange réactionnel a été préparé en mélangeant $1 \mathrm{ml}$ de solution d'extrait de $0,5 \mathrm{ml}$ de réactif de Folin-Ciocalteu $(0,5 \mathrm{~N})$ et $1,5 \mathrm{ml}$ de carbonate de sodium $(\mathrm{NaHC03})$ à $17 \%$. L'ensemble est incubé à $37{ }^{\circ} \mathrm{C}$ pendant $30 \mathrm{~min}$ et l'absorbance est mesurée à $760 \mathrm{~nm}$ contre un blanc sans extrait pris comme référence. La quantification des composés phénoliques totaux est calculée à partir d'une droite d'étalon 
de l'acide gallique $(0-1000 \mu \mathrm{g} / \mathrm{ml})$ et exprimé en $\mu \mathrm{g}$ équivalent d'acide gallique (EAG/g MS).

Le dosage des flavonoïdes totaux a été fait selon la méthode modifiée de Hariri et al. (1991). A $2 \mathrm{ml}$ de chaque extrait, sont ajoutés $100 \mu 1$ de réactif de Neu. L'absorbance est déterminée à $404 \mathrm{~nm}$. La teneur en flavonoïdes est exprimée en pourcentage (\%).

\section{Dosage de l'activité antioxydante}

L'évaluation quantitative du potentiel antioxydant des extraits a été réalisée in vitro par le test au DPPH (1,1-diphényl-2-picrylhydrazyle). Le protocole est celui décrit par (Blois, 1958) avec de légères modifications. 1 $\mathrm{ml}$ d'extrait éthanolique, à différentes gammes de concentration et $1,5 \mathrm{ml}$ d'une solution éthanolique de DPPH $(0,03 \mathrm{mg} / \mathrm{ml})$ ont été soigneusement mélangés et conservés à l'obscurité pendant $30 \mathrm{~min}$. Le blanc a été préparé en remplaçant l'extrait par l'éthanol $(1 \mathrm{ml})$. L'absorbance du mélange réactionnel a été mesurée à $517 \mathrm{~nm}$. Le pourcentage d'inhibition du DPPH est calculé suivant la formule :

$$
\text { PI \% }=\left[1-\left(\mathrm{A}_{e} / \mathrm{A}_{b}\right)\right] \times 100
$$

$\mathrm{A}_{\mathrm{e}}$ : absorbance de l'échantillon ;

$\mathrm{A}_{\mathrm{b}}$ : absorbance du blanc.
Les valeurs de $\mathrm{CI}_{50}$ ont été estimées à partir de la courbe de pourcentage d'inhibition par rapport à la concentration.

\section{Toxicité aigüe des extraits}

Les essais de toxicité aigüe ont été menés suivant la méthode de «l'ajustement des doses » de la ligne 425 de l'OCDE (OCDE, 2008) et a consisté à tester les extraits bruts en solution aqueuse, à 2000 et $5000 \mathrm{mg} / \mathrm{kg}$ de poids corporel (pc). La dose d'essai limite de $5000 \mathrm{mg} / \mathrm{kg}$ de pc a été utilisée pour déterminer la $\mathrm{DL}_{50}$. La dose de $2000 \mathrm{mg} / \mathrm{kg}$ de pc était l'essai préliminaire. L'évaluation de la toxicité a été réalisée par voie orale, sur 11 lots de trois rattes WISTAR, saines, nullipares et non gravides, âgées de 09 semaines dont les poids variaient de 52 à $86 \mathrm{~g}$. Avant les expériences, les animaux ont été acclimatés aux conditions de laboratoire. Les extraits ont été administrés par voie orale, à l'aide d'une sonde à gavage, le lot témoin recevant de l'eau distillée. La dose de $5000 \mathrm{mg} / \mathrm{kg}$ de pc correspondait à 500 $\mathrm{mg} / \mathrm{ml}$ des extraits aqueux pour une ratte pesant 100 g. Les animaux ont été observés durant 14 jours. Les signes cliniques et les mortalités observés ont été notés.

Tableau 1 : Plantes sélectionnées pour les tests phytochimiques, antioxydants et de toxicité.

\begin{tabular}{lcc}
\hline Espèces & Famille & Parties utilisées \\
\hline Ficus mucuso Ficalho & Moraceae & Ecorces \\
Ziziphus mauritiana Lam. & Rhamnaceae & Rameaux feuillés \\
Ocimum gratissimum L. & Lamiaceae & Feuilles \\
Catharanthus roseus (L.) G.Don & Apocynaceae & Plante entière \\
Tamarindus indica L. & Fabaceae & Ecores de tige et Feuilles \\
\hline
\end{tabular}




\section{RESULTATS \\ Criblage phytochimique des extraits végétaux}

Le criblage phytochimique réalisé a permis d'observer les polyphénols, les tanins et les flavonoïdes dans les extraits aqueux de feuilles de Ocimum gratissimum, de plante entière de Catharanthus roseus, d'écorces de tige de Fucus mucuso, du mélange écorces de tige/feuilles de Tamarindus indica, de plante entière de Catharanthus roseus et des rameaux feuillés de Ziziphus mauritiana (Tableau 2). Les stérols sont présents uniquement dans l'extrait aqueux de plante entière de Catharanthus roseus. Les alcaloïdes sont présents dans les extraits aqueux de rameaux feuillés de Ziziphus mauritiana et de plante entière de Catharanthus roseus. Les saponosides sont présents dans les extraits aqueux de plante entière de Catharanthus roseus, de feuilles de Ziziphus mauritiana et du mélange écorces de tige/feuilles de Tamarindus indica. L'extrait aqueux de rameaux feuillés de Ziziphus mauritiana a présenté 5 phytocomposés sur 6 recherchés.

\section{Dosages des polyphénols totaux et flavonoïdes totaux}

Le dosage des polyphénols a montré que les teneurs observées dans les différents extraits étudiés (Tableau 3). Ils varient très significativement ( $\alpha=0,05 ; P<0,0001)$, entre 18000 et $84000 \mu \mathrm{g}$ EAG/g MS. Les taux les plus élevés ont été observés dans les extraits aqueux d'écorce de tige de Ficus mucuso $(83425,29 \mu \mathrm{g}$ EAG/g MS) et de rameaux feuillés de Zizyphus mauritiana (74517,24 $\mu \mathrm{g}$ EAG/g MS). Les extraits aqueux du mélange écorces de tige/feuilles de Tamarindus indica et des feuilles Ocimum gratissimum ont présentés respectivement 34747,13 et 23454,02 $\mu \mathrm{g}$ EAG/g MS. L'extrait aqueux de la plante entière de Catharanthus roseus a présenté la plus faible teneur en polyphénols totaux.

Les teneurs en flavonoïdes totaux (Tableau 4), comme celles des polyphénols totaux, varient également significativement ( $\alpha$ $=0,05 ; P<0.0001)$ d'un extrait à l'autre. La plus forte teneur en flavonoïdes a été observée avec l'extrait aqueux de rameaux feuillés de Ziziphus mauritiana $(20,28 \%)$ et la plus faible avec l'extrait aqueux d'écorce de tige de Ficus mисиso $(6,81 \%)$. Les valeurs intermédiaires sont obtenues avec les extraits aqueux du mélange écorces de tige/feuilles de Tamarindus indica (14,46\%), de feuilles d'Ocimum gratissimum $(12,38 \%)$ et de plante entière de Catharanthus roseus $(12,35 \%)$.

\section{Activité antioxydante}

Les résultats de l'étude de l'activité antioxydante des différents extraits de plantes étudiés ont montré que l'extrait aqueux du mélange écorces de tige/feuilles de Tamarindus indica est présente les meilleurs pourcentages d'inhibition des radicaux libres, entre 12,98 et $95,7 \%$, à des concentrations variant de 0,0032 à $0,5 \mathrm{mg} / \mathrm{ml}$ d'extraits bruts (Figure 1), suivi de l'extrait aqueux d'écorce de tige de Ficus mucuso (avec des concentrations variant de 0,0156 à $0,5 \mathrm{mg} / \mathrm{ml}$ les pourcentages d'inhibition varient de 12,58 à 92,76\%). Pour les mêmes concentrations, les extraits aqueux de rameaux feuillés de Ziziphus mauritiana, de la plante entière de Catharanthus roseus et des feuilles de Ocimum gratissimum ont présenté des pourcentages d'inhibition qui varient, respectivement, entre 13.90 et $87,00 \%, 2,53$ et $90,24,3,74$ et 82,35 . Les pourcentages d'inhibition de $50 \%$ des radicaux libres $\left(\mathrm{CI}_{50}\right)$ par les différents extraits aqueux varient très significativement ( $\alpha=0,05 ; P<0.0001)$ d'un extrait à l'autre. Les $\mathrm{CI}_{50}$ varient de $0,1269 \pm$ 0,0004 à $0,2319 \pm 0,0002 \mathrm{mg} / \mathrm{ml}$. L'activité anti-radicalaire (Tableau 5) montre que les capacités antioxydantes les plus importantes sont observées avec les extraits aqueux du mélange écorces de tige/feuilles de Tamarindus indica, avec une $\mathrm{CI}_{50}=0,1269 \pm$ $0,0004 \mathrm{mg} / \mathrm{ml}$ et de ceux des écorces de tige de Ficus muсuso $(0,1335 \pm 0,0004 \mathrm{mg} / \mathrm{ml})$.

\section{Toxicité des extraits aqueux sur des rats}

Les tests de toxicité aiguë réalisés à partir des extraits bruts en solution aqueuse montrent des signes de toxicité aiguë à la dose de $5000 \mathrm{mg} / \mathrm{kg}$ de pc pour l'extrait aqueux des écorces de tige de Ficus mucuso. Elle a provoqué des tremblements, des convulsions et 
la mortalité chez un (1) rat. Pour les autres extraits aqueux testés, aucune mortalité et aucun signe clinique n'a été observé aux différentes doses, $2000 \mathrm{mg} / \mathrm{kg}$ de pc et de 5000 $\mathrm{mg} / \mathrm{kg}$ de pc (Tableau 6). Tous les animaux gavés ont survécu après les 14 jours d'observation. Les extraits bruts aqueux des rameaux feuillés de Zizifus mauritiana, des feuilles de Ocimum gratissimum, de la plante entière de Catharanthus roseus, du mélange écorces de tige/feuilles de Tamarindus indica, administrés par gavage sont non toxiques chez des rats Wistar selon le Système Général Harmonisé de classification et d'étiquetage des produits chimiques (SGH, 2003). L'extrait aqueux des écorces de tige de Ficus mucuso est le seul à présenter une toxicité aigüe chez le rat Wistar à $5000 \mathrm{mg} / \mathrm{kg}$ de pc. Cependant, à 2000 $\mathrm{mg} / \mathrm{kg}$ de pc, aucune mortalité n'a été observée.

Tableau 2 : Résultats des tests phytochimiques.

\begin{tabular}{llcccccc}
\hline & & \multicolumn{7}{c}{ Métabolites recherchés } \\
\hline Extraits & Partie de plantes & Tan & Poly & Flav & Stér & Sap & Alc \\
\hline Catharanthus roseus & Plante entière & ++ & +++ & + & ++ & - & - \\
Ficus mucuso & Ecorces de tige & +++ & +++ & +++ & - & - & - \\
Ocimum gratissimum & Feuilles & ++ & +++ & + & - & ++ & - \\
Tamarindus indica & Ec. de tige et Feuilles & + & + & ++ & - & +++ & - \\
Ziziphus mauritiana & Rameaux feuillés & +++ & +++ & ++ & - & +++ & ++ \\
\hline
\end{tabular}

Signification des symboles : Tan : Tanins ; Poly : Polyphénols ; Flav : Flavonoïdes ; Stér : Stérols ; Sap : Saponosides ; Alc :

Alcaloïdes ; Ec. : Ecorce ; + : Présent ; ++ : Abondant ; +++ : Très abondant ; - : Absent.

Tableau 3 : Teneur en polyphénols totaux des extraits aqueux de plantes.

\begin{tabular}{llllll}
\hline Extraits & Partie de plante & $\begin{array}{l}\text { Teneurs } \\
(\boldsymbol{\mu g} \text { EAG/g MS }) \pm \text { SD }\end{array}$ & dl & $\boldsymbol{F}$ & $\boldsymbol{P}$ \\
\hline Ficus mucuso & Ecorces de tige & $83425,29 \pm 9563^{\mathrm{c}}$ & & & \\
Ziziphus mauritiana & Rameaux feuillés & $74517,24 \pm 2134^{\mathrm{c}}$ & & & \\
Tamarindus indica & Ec. de tige et Feuilles & $34747,13 \pm 179^{\mathrm{ef}}$ & 4 & 85.97 & 0.0001 \\
Oocimum gratissimum & Feuilles & $23454,02 \pm 2126^{\mathrm{fg}}$ & & & \\
Catharanthus roseus & Plante entière & $19718,39 \pm 1466^{\mathrm{fg}}$ & & & \\
\hline
\end{tabular}

$\mathrm{dl}$ : degré de liberté $; F=$ test Fisher ; $P=p$-value $<0,05$ indique une différence significative entre les différents extraits.

Tableau 4 : Teneur en flavomoïdes des extraits aqueux de plantes.

\begin{tabular}{llllll}
\hline Extraits & Partie de plante & \% Flavonoïdes & dl & $\boldsymbol{F}$ & $\boldsymbol{P}$ \\
\hline Ziziphus mauritiana & Rameaux feuillés & $20,28 \pm 1,97^{\mathrm{bc}}$ & & & \\
Tamarindus indica & Ec. de tige et Feuilles & $14,46 \pm 0,68^{\mathrm{d}}$ & & & \\
Ocimum gratissimum & Feuilles & $12,38 \pm 1,09^{\mathrm{e}}$ & 4 & 1508 & 0,0001 \\
Catharanthus roseus & Plante entière & $12,35 \pm 0,75^{\mathrm{e}}$ & & & \\
Ficus mucuso & Ecorces de tige & $6,81 \pm 1,75^{\mathrm{f}}$ & & & \\
\hline
\end{tabular}




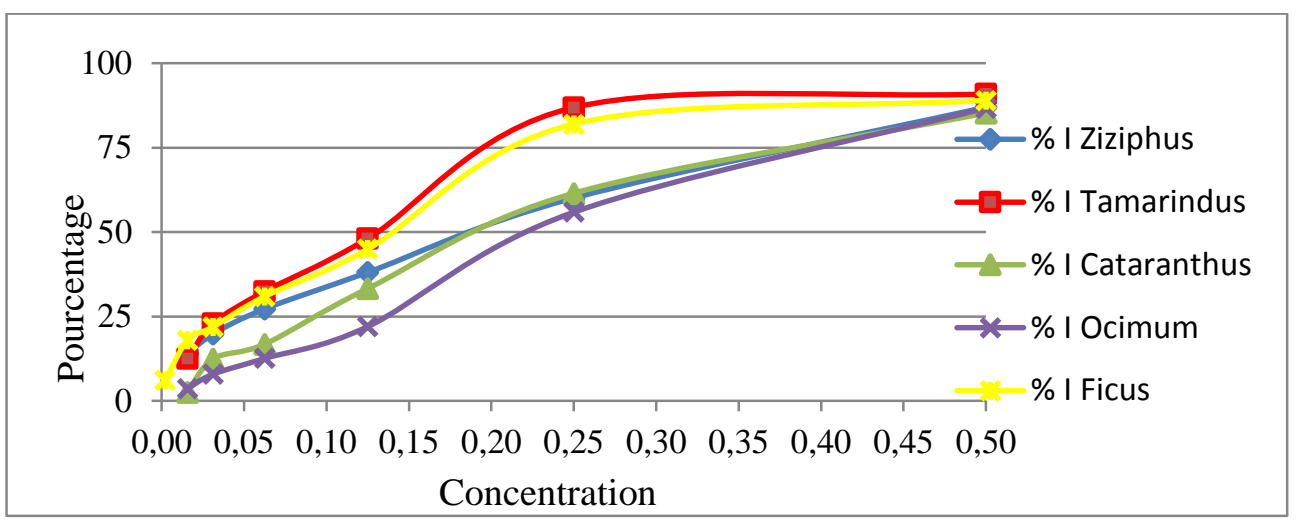

Figure 1 : Activités antioxydantes des différents extraits aqueux des plantes.

Tableau 5 : Concentrations inhibitrices $50\left(\mathrm{CI}_{50}\right)$ des extraits aqueux de plantes.

\begin{tabular}{llllll}
\hline Extraits & Partie de plante & CI50 $(\mathbf{m g} / \mathbf{m L}) \pm$ SD & dl & $\boldsymbol{F}$ & $\boldsymbol{P}$ \\
\hline Vit C & & $0,0054 \pm 0,0002^{\mathrm{a}}$ & & & \\
Tamarindus indica & Ec. de tige et Feuilles & $0,1269 \pm 0,0004^{\mathrm{d}}$ & & & \\
Ficus mucuso & Ecorces de tige & $0,1335 \pm 0,0004^{\mathrm{d}}$ & 4 & 10210 & $<0,0001$ \\
Ziziphus mauritiana & Rameaux feuillés & $0,2246 \pm 0,001^{\mathrm{e}}$ & & & \\
Catharanthus roseus & Plante entière & $0,2312 \pm 0,0003^{\mathrm{e}}$ & & & \\
Ocimim gratissimum & Feuilles & $0,2319 \pm 0,0002^{\mathrm{e}}$ & & & \\
\hline \multicolumn{1}{c}{ dl $:$ degré de liberté $; F=$ test Fisher $\cdot P=p$-value $<0,05$ indique une différence significative entre les différents extraits. }
\end{tabular}

Tableau 6 : Tests de toxicité aiguë des différents extraits aqueux.

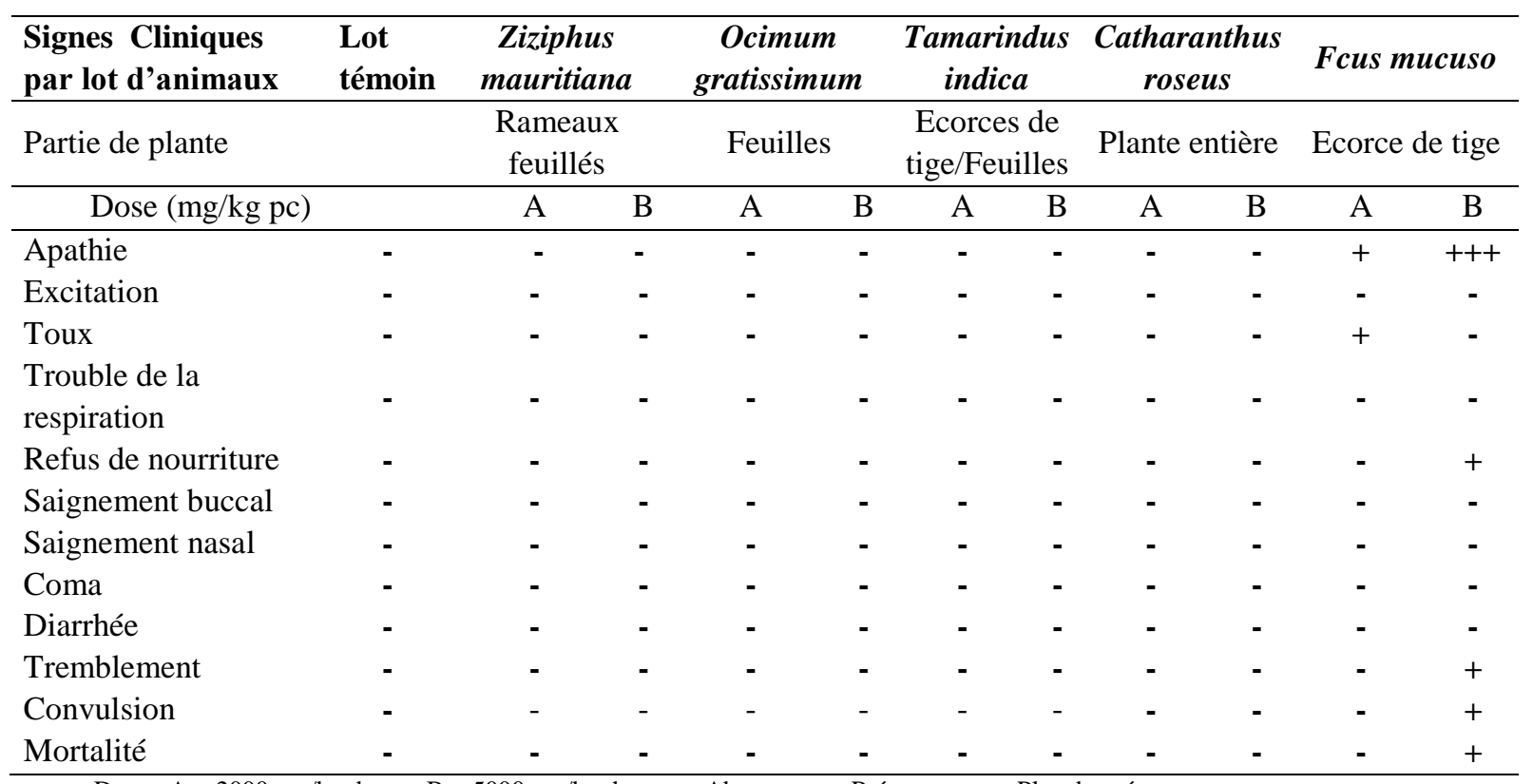




\section{DISCUSSION}

L'analyse phytochimique qualitative des extraits totaux des plantes retenues pour la présente étude a mis en évidence la richesse des extraits aqueux en tanins, en polyphénols totaux, en flavonoïdes et, pour certains, en alcaloïdes. Ces résultats confortent l'utilisation de ces drogues dans le traitement des séquelles des AVC et dans la prévention secondaire des facteurs de risque. Les travaux de Dongock et al. (2018), au Tchad, indiquent la richesse en tanins, flavonoïdes, alcaloïdes et saponines des plantes utilisées dans le traitement des maladies cardiovasculaires. Ces métabolites secondaires ont des propriétés très reconnues dans le traitement et la prévention des maladies cardiovasculaires. En effet les études de Kee et al. (2008) et de Praveen et Kumud (2012) ont montré que les tanins présentent des propriétés antithrombotiques et anticoagulantes. Selon les travaux de Sereme et al. (2008), les drogues végétales riches en tanins sont spécifiquement efficaces dans le traitement de l'hypertension artérielle. De même, ceux de Chen et al. (2009), puis O'Leary et al. (2004) indiquent que les flavonoïdes présentent des activités antiinflammatoires, antihypertensives (antiallergiques) et antiplaquettaires, respectivement. Quant aux travaux de Proteggente et al. (2011), puis Luna-Vázquez et al. (2013), ils montrent que les polyphénols présentent des propriétés vasodilatatrices et antihypertensives, en plus des propriétés antithrombotiques et anti-inflammatoires (Manach et al., 2005). Les saponines dans les plantes médicinales sont responsables de la plupart des effets biologiques liés à la croissance et à la division des cellules chez les humains et peuvent avoir un effet analgésique et antiinflammatoire (Chindo et al., 2010). Ils pourraient donc favoriser une régénération des cellules nerveuses.

La grande majorité de ces études ont déjà montré les propriétés antioxydantes des tanins, des flavonoïdes et des polyphénols. Ces composés connus pour leur capacité de piégeage des radicaux libres (Kanfon et al., 2018) peuvent prévenir le développement de nombreuses maladies chroniques (Vadivel et al., 2011). Ils sont indispensables pour réduire le stress oxydatif après un AVC, puis permettre une régénération du tissu cérébral, résorber la lésion et donc les déficits moteurs (Quintard, 2016). Plusieurs études chez des modèles animaux et chez des sujets humains ont effectivement confirmé la biodisponibilité des phénols à exercer un rôle protecteur contre le stress oxydatif et les dommages des radicaux libres (Prior, 2003 ; Szajdek et al., 2008).

L'analyse phytochimique quantitative réalisée a montré que les extraits aqueux des écorces de tige de Ficus mucuso, des rameaux feuillés de Zizuplus mauritiana et du mélange écorces de tige/feuilles de Tamarindus indica ont présenté les plus fortes teneurs en polyphénols et en flavonoïdes. Ces extraits aqueux ont présenté également les capacités antioxydantes les plus importantes avec, respectivement, une $\mathrm{CI}_{50}$ de $0,12 \mathrm{mg} / \mathrm{ml}$ pour le mélange écorces de tige/feuilles de Tamanrindus indica, $0,13 \mathrm{mg} / \mathrm{ml}$ pour l'extrait aqueux des écorces de tige de Ficus mисиso et 2,22 $\mathrm{mg} / \mathrm{ml}$ pour les rameaux feuillés de Ziziphus mauritiana. Ces extraits de plantes présentent les potentiels les plus élevés pour le traitement des séquelles des AVC. L'utilisation des molécules végétales à haut pouvoir antioxydant est une des solutions pour lutter contre l'oxydation (Rolland, 2004). Le pouvoir antioxydant est indispensable dans le traitement des séquelles des AVC par sa capacité à réduire le stress oxydant et à favoriser la régénération des cellules du cerveau (Quintard, 2014). Le potentiel antioxydant des drogues est, entre autre une donnée non négligeable dans la recherche sur les molécules anti-oxydantes.

L'étude de la toxicité aiguë par voie orale chez les rats Wistar n'a présenté aucun mort et aucun signe clinique de toxicité au terme des 14 jours, aux doses comprises entre 2000 et $5000 \mathrm{mg} / \mathrm{kg}$ de $\mathrm{pc}$ avec les extraits aqueux des rameaux feuillés de Ziziphus mauritiana, de la plante entière de Catharanthus roseus, des feuilles de Ocimim gratissimum et du mélange écorces de tige/feuilles de Tamarindus indica. Ces quatre plantes très utilisées dans le traitement des symptômes des AVC (Piba et al., 2018) ont donc une $\mathrm{DL}_{50}$ supérieure à $5000 \mathrm{mg} / \mathrm{kg}$ de pc. 
Selon le système de classification globalement harmonisé de l'OCDE (OCDE, 2001), ces extraits totaux aqueux sont classés dans la catégorie 5 et considérés comme des substances non toxiques par voie orale. L'extrait aqueux des écorces de tige de Ficus тисияо a montré des signes cliniques tels que des tremblements, des convulsions et une mortalité à $5000 \mathrm{mg} / \mathrm{kg}$ de pc. Selon l'échelle de toxicité de Hodge et Sterner (1949), cet extrait aqueux, administré par voie orale n'est pas toxique à $2000 \mathrm{mg} / \mathrm{kg}$ de pc et légèrement toxique par voie orale, chez les souris, à 5000 $\mathrm{mg} / \mathrm{kg}$ de pc dans les conditions de notre étude. Cet organe de plante devrait faire l'objet d'une attention particulière et devrait être utilisé à une dose inférieure à $2000 \mathrm{mg} / \mathrm{kg}$ de $\mathrm{pc}$. La dose de $2000 \mathrm{mg} / \mathrm{kg}$ de pc, qui apparait comme la plus élevée tolérée par l'organisme du rat, devrait cependant être utilisée à titre expérimentale dans une étude de toxicité subaiguë ou chronique pour évaluer son innocuité.

\section{Conclusion}

La présente étude a porté sur l'évaluation de la toxicité aiguë, l'analyse phytochimique et l'activité antioxydante de 5 extraits de plantes utilisées dans le traitement des séquelles de des AVC en Côte d'Ivoire. Les résultats de l'étude de la toxicité aigüe par voie orale ont montré que les extraits aqueux des rameaux feuillés de Ziziphus jujuba, de la plante entière de Catharanthus roseus, des feuilles de Ocimim gratissimum et du mélange écorces de tige/feuilles de Tamarindus indica, utilisés dans le traitement des séquelles des AVC sont classés dans la catégorie 5 et considérés non toxique par voie orale. Seul l'extrait aqueux des écorces de tige de Ficus mucuso a présenté une létalité à $5000 \mathrm{mg} / \mathrm{kg}$ de pc. Il est donc qualifié de légèrement toxique par voie orale. L'analyse phytochimique qualitative des extraits totaux a mis en évidence leur richesse en tanins, en polyphénols totaux, en flavonoïdes et pour certains, en alcaloïdes. Ces résultats confortent l'utilisation de ces drogues dans le traitement des séquelles des AVC et dans la prévention secondaire des facteurs de risque. Les extraits aqueux du mélange écorces de tige/feuilles de
Tamarindus indica et des écorces de tige de Ficus тисиso ont présenté de bonnes capacités antioxydantes avec respectivement des $\mathrm{CI}_{50}$ de 0,12 et $0,13 \mathrm{mg} / \mathrm{ml}$ d'extrait brut. Ces résultats sont prometteurs dans la recherche de traitements efficaces pour la prise en charge des malades victimes des AVC. Des investigations pharmacologiques plus poussées permettront de déterminer l'efficacité de ces extraits in vivo des extraits de Tamarindus indica et des écorces de tige de Ficus mucuso.

\section{CONFLIT D'INTÉRÊTS}

Les auteurs déclarent qu'ils n'ont aucun conflit d'intérêts.

\section{CONTRIBUTIONS DES AUTEURS}

SCP : Enquête ethnomédecine, analyse et rédaction; PAKK : Tests phytochimiques, tests antioxydants; LNK : Enquête ethnomédecine, tests phytochimiques; RKDK: Tests phytochimiques, tests de toxicité ; AGK: Tests phytochimiques, tests de toxicité ; HFBT: Rédaction, Superviseur Scientifique.

\section{REMERCIEMENTS}

Les auteurs de ce manuscrit remercient l'ASCAD (Académie des Sciences, des Arts, des Cultures d'Afrique et des Diasporas Africaines) pour sa contribution financière à cette étude, le Programme National de Promotion de la Médecine Traditionnelle en Côte d'Ivoire, tous les phytothérapeutes et herboristes pour leur disponibilité.

\section{REFERENCES}

Abboud H, Amarenco P. 2004. Thrombolyse et infarctus cérébral aigu. MEDECINE/SCIENCES, 12(20): 11041108. DOI : http://dx.doi.org/10.1051/medsci/200420 121104

Agbodan KA, Dotse K et Koumaglo KH. 2014. Activités antioxydantes des huiles essentielles de trois plantes aromatiques acclimatées au Togo. Int. J. Biol. Chem. Sci., 8(3): 1103-1110. DOI: http://dx.doi.org/10.4314/ijbcs.v8i3.23 
Blois M. 1958. Déterminations antioxydantes par l'utilisation d'un radical libre stable. Nature, 181: 1199-1200. DOI: https://doi.org/10.1038/1811199a0

Bordet R, Ouk T, Onteniente B, CharriautMarlangue C, Heurteaux C. 2009. Ischemie cerebrale. Les pistes therapeutiques de demain. Med. Sci. (Paris), 25: 847-854. DOI: 10.1051/medsci/20092510847

Chen J, Du CY, Lam KY, Zhang WL, Lam CT, Yan AL, Yao P, Lau DT, Dong TT, Tsim KW. 2014. The standardized extract of Ziziphus Jujuba fruit (jujube) regulates pro-inflammatory cytokine expression in cultured murine macrophages: suppression of lipopolysaccharidestimulated NF-kB activity. Phytother Res., 28(10): 1527-1532. DOI: https://doi.org/10.1002/ptr.5160

Chindo BA, Anuka JA, Isaac E, Ahmadu AA, Tarfa DF, Gamaniel KS. 2010. Saponins are involved in the analgesic and antiinflammatory properties of Ficus platyphylla stem bark. Int. J. Biol. Chem. Sci., 4(2): 415-423. DOI: 10.4314/ijbcs.v4i2.58140

Dongock DN, Bonyo LA, Mapongmestem MP, Bayegone E. 2018. Etude ethnobotanique et phytochimique des plantes médicinales utilisées dans le traitement des maladies cardiovasculaires à Moundou (Tchad). Int. J. Biol. Chem. Sci., 12(1): 203-216. DOI:

https://dx.doi.org/10.4314/ijbcs.v12i1.16

Evenamede KS, Kpegba K, Simalou O, Boyode P, Agbonon A, Gbeassor M. 2017. Etude comparative des activités antioxydantes d'extraits éthanoliques de feuilles, d'écorces et de racines de Cassia sieberiana. Int. J. Biol. Chem. Sci., 11(6): 2924-2935.

DOI:

https://dx.doi.org/10.4314/ijbcs.v11i6.29

Feigin VL, Forouzanfar MH, Krishnamurthi R, Mensah GA, Connor M, Bennett DA, Moran AE, Sacco RL, Anderson L, Truelsen T, O'Donnell M, Venketsubramanian N, Barker-Collo S, Lawes CMM, Wang W, Shinohara Y,
Witt E, Ezzati M, Naghavi M, Murray C. 2014. Global and regional burden of stroke during 1990-2010: findings from the Global Burden of Disease Study 2010. Lancet, 383(9913): 245-255. DOI: 10.1016/s0140-6736(13)61953-4

Go AS, Mozaffarian D, Roger VL, Benjamin EJ, Berry JD, Blaha MJ, Dai S, Ford ES, Fox CS, Franco S, Fullerton HJ, Gillespie C, Hailpern SM, Heit JA, Howard VJ, Huffman MD, Judd SE, Kissela BM, Kittner SJ, Lackland DT, Lichtman JH, Lisabeth LD, Mackey RH, Magid DJ, Marcus GM, Marelli A, Matchar DB, McGuire DK, Mohler ER, Moy CS, Mussolino ME, Neumar RW, Nichol G, Pandey DK, Paynter NP, Reeves MJ, Sorlie PD, Stein J, Towfighi A, Turan $\mathrm{TN}$, Virani SS, Wong ND, Woo D, Turner MB. 2014. Heart disease and stroke statistics--update: a report from the american heart association. Circulation, 129(3): 28-292. DOI: https://doi.org/10.1161/01.cir.000044113 9.02102 .80

Hariri EB, Sallé G, Andary C. 1991. Involvement of flavonoids in the resistance of two popular cultivars to mistletoe (Viscum album L.). Protoplasma, 162(1): 20-26. DOI: 10.1007 / BF01403897

Harbome JB. 1998. Phytochemical methods. In A Guide to Modern Techniques of Plant Analysis (3rd edn). Chapman and Hall: New York, NY: 40-137.

Hacke W, Kaste M, Bluhmki E, Brozman M, Dávalos A, Guidetti D, Larrue V, Lees KR, Medeghri Z, Machnig T, Schneider D, von Kummer R, Wahlgren N, Toni D. 2008. Thrombolysis with alteplase 3 to 4.5 hours after acute ischemic stroke. $N$. Engl. J. Med., 359: 1317-29. DOI: https://doi.org/10.1056/NEJMoa0804656

HAS. 2012. Accident vasculaire cérébral : méthodes de rééducation de la fonction motrice chez l'adulte Méthode " Recommandations pour la pratique clinique ». Autorité de Santé, 21 p. www.has-sante.fr 
HAS. 2015. Fiche descriptive AVC. Haute Autorité de Santé, 18 p. www.has-sante.fr Heilerová L, Bučková M, Tarapčík P, Šilhár S, Labuda J. 2003. Comparaison des données d'activité antioxydante pour les extraits aqueux de mélisse (Melissa officinalis L.), d'origan (Origanum vulgare $\mathrm{L}$.), le thym (Thymus vulgaris $\mathrm{L}$.) et l'agrimonie (Agrimonia eupatoria L.) obtenus par des méthodes conventionnelles et le biocapteur à base d'ADN. Czech J. Food Sci., 21: 78-84. DOI: https://doi.org/10.17221/3480CJFS

Hodge HC, Sterner JH. 1949. Tabulation of Toxicity Classes. American Industrial Hygiene Association Quarterly, 10(4): 93-96.

DOI: 10.1080/00968204909344159

Just MJ, Recio MC, Giner RM, Cuellar MJ, Manez S, Bilia AR, Rios JL. 1998. Antiinflammatory activity of unusual lupine saponins from Bupleurum fruticescens. Plant. Med., 64: 404-407. DOI: $10.1055 / \mathrm{s}-2006-957469$

Kanfon RE, Gnawe M, Dossa ACP, Yedomonhan H, Wot To DV, Sohounhloue CKD. 2018. Caractérisation physico-chimique et évaluation de l'activité antiradicalaire des extraits de sept morphotypes de gombo (Abelmoschus spp.) cultivés au Bénin. Int. J. Biol. Chem. Sci., 12(3): 1447-1458. DOI:

https://dx.doi.org/10.4314/ijbcs.v12i3.30

Kee NLA, Mnonopi N, Hajierah D, Naudé RJ, Frost CL. 2008 . Antithrombotic/anticoagulant and anticancer activities of selected medicinal plants from South Africa. African Journal of Biotechnology, 7(3): 217-223. DOI: https://doi.org/10.5897/AJB07.834

Lamblin A, Bascou M, Drouard E, Alberti N, De Greslan T. 2018. Thrombolytic treatment of vertebro-basilar ischemic stroke in N'Djamena, Republic of Chad. The Pan African Medical Journal, Case report, 5p. DOI: 10.11604/pamj.2018.29.35.14547
Leys D, Cordonnier C. 2009. Traitements des accidents vasculaires cérébraux en phase aigüe et prévention secondaire. Med. Sci., 25: 733-738.

DOI : https://doi.org/10.1051/medsci/20092589733

Luna-Vázquez FJ, Ibarra-Alvarado C, RojasMolina A, Rojas-Molina JI, Yahia EM, Rivera-Pastrana DM, Rojas-Molina A, Zavala-Sánchez MA. 2013. Nutraceutical Value of Black Cherry Prunus serotina Ehrh. Fruits: Antioxidant and Antihypertensive Properties. Molecules, 18: 14597-14612. DOI:10.3390/molecules 181214597

Manach C, Williamson G, Morand C, Scalbert A, Remesy C. 2005. Bioavailability and bioefficacy of polyphenols in humans. I. Review of 97 bioavailability studies. Am. J. Clin. Nutr., 81: 230S-242S. DOI: https://doi.org/10.1093/ajcn/81.1.230S

Masingue M, Alamowitch S. 2015. Nouvelles limites de la thrombolyse intraveineuse dans le traitement des infarctus cérébraux. La Presse Médicale, 4(5): 515-525. http://dx.doi.org/10.1016/jlpm.2014.07.0 27

OCDE. 2001. Sous-comité d'experts du système général harmonisé de classification et d'étiquetage des produits chimiques : dangers pour la santé et l'environnement - toxicité aiguë. UN/SCEGHS/2/INF.11, 13p.

OCDE. 2008. Pharmacopée Européenne (6 $6^{\mathrm{eme}}$ édn, Tome 1). OCDE ; 178-568.

O’Leary KA, Pascual-Tereasa S, Needs PW, Bao YP, O'Brien NM, Williamson G. 2004. Effect of flavonoids and vitamin $\mathrm{E}$ on cyclooxygenase-2 (COX-2) transcription. Muta. Res. Fund. Mol. Mech. Mut., 551: 245-254. DOI: 10.1016/j.mrfmmm.2004.01.015

Piba SC, Kouakou DKR, Kouamé AG, Koné MW, Bakayoko A, Tra Bi FH. 1998. Ethnobotanical studies of medicinal plants used in the treatment of stroke sequelae in the district of Abidjan, Côte d'Ivoire. Int. J. Adv. Res., 6(10), 90-102. DOI: 10.21474/IJAR01/7975 
Praveen K, Kumud U. 2012. Tannins are Astringent. Journal of Pharmacognosy and Phytochemistry, 1(3): 45-50. DOI :

https://www.phytojournal.com/archives/ 2012/vol1issue3/PartA/8.1.pdf

Proteggente AR, Pannala AS, Paganga G, van Buren L, Wagner E, Wiseman S, Van de Put F, Dacombe C, Rice-Evans C. 2002. The antioxidant activity of regularly consumed fruit and vegetables reflects their phenolic and vitamin $\mathrm{C}$ composition. Free Radic. Res., 36: 217-233. DOI : https://doi.org/10.1080/10715760290006 484

Quintard H. 2014. Etude des propriétés neuroprotectrices et neurorégénératives du MLC901, issu de la Médecine Traditionnelle Chinoise face à l'ischémie globale et au traumatisme crânien chez le rongeur. Sciences agricoles. Université Nice Sophia Antipolis, 206 p.

Prior RL. 2003. Fruits and vegetables in the prevention of cellular oxidative damage. Am. J. Clin. Nutr., 78: 570S-578S. DOI: https://doi.org/10.1093/ajcn/78.3.570S

Ramya D, Thirunavukkarasu P, Barathi A, Asha S. 2017. In vitro anticoagulant activity of Nelumbo nucifera leaf extracts on normal healthy blood plasma. International Journal of Green Pharmacy, 11(3): 16. DOI: http://dx.doi.org/10.22377/ijgp.v11 i03.1121

Rolland Y. 2004. Antioxydants naturels végétaux. OCL., 11(6): 419-424. DOI: 10.1051/ocl.2004.0419
Sereme A, Millogo-Rasolodimby J, Guinko S, Nacro M. 2008. Propriétés thérapeutiques des plantes a tanins du Burkina Faso. Pharmacopée et Médecine Traditionnelle Africaines, 15: 41-49. DOI: http://publication.lecames.org/index.php/ pharm/article/view/8/7

Singleton V, Orthofer R, Lamuela-raventos RM. 1999. Analysis of total phenols and other oxidation substrates and antioxidants by means of Folin-Ciocalteu reagent. Methods Enzymol, 299: 152-178. DOI: https://doi.org/10.1016/S00766879(99)99017

Sutherland BA, Minnerup J, Balami JS, Arba F, Buchan AM, Kleinschnitz C. 2012. Neuroprotection for ischaemic stroke: Translation from the bench to the bedside. Int. J. Stroke, 7: 407-418. DOI: https://doi.org/10.1111/j.17474949.2012.00770.x

Szajdek A, Borowska EJ. 2008. Bioactive compounds and health-promoting properties of berry fruits: A review. Plant Foods Hum. Nutr., 63: 147-156. DOI: 10.1007/s11130-008-0097-5

Vadivel V, Biesalski HK. 2011. Contribution of phenolic compounds to the antioxidant potential and type II diabetes related enzyme inhibition properties of Pongamia pinnata L. Pierre seeds. Process Biochem., 46(10): 1973-1980. DOI:

https://doi.org/10.1016/j.procbio.2011.07 .007 